\title{
Magnetic field dependence of atomic collapse in bilayer graphene
}

\author{
R. Van Pottelberge,,$^{1, *}$ M. Zarenia,,${ }^{1,2, \dagger}$ and F. M. Peeters ${ }^{1, \ddagger}$ \\ ${ }^{1}$ Departement Fysica, Universiteit Antwerpen, Groenenborgerlaan 171, B-2020 Antwerpen, Belgium \\ ${ }^{2}$ Department of Physics and Astronomy, University of Missouri, Missouri, Columbia 65211, USA
}

(Received 9 April 2018; revised manuscript received 21 June 2018; published 4 September 2018)

\begin{abstract}
The spectrum of a Coulomb impurity in bilayer graphene is investigated as function of the strength of a perpendicular magnetic field for different values of the angular quantum number $m$ and for different values of the gate voltage. We point out fundamental differences between the results from the two-band and four-band model. The supercritical instability and fall-to-center phenomena are investigated in the presence of a magnetic field. We find that in the four-band model the fall-to-center phenomenon occurs as in monolayer graphene, while this is not the case in the two-band model. We find that in a magnetic field the supercritical instability manifests itself as a series of anticrossings in the hole part of the spectrum for states coming from the low-energy band. However, we also find very distinct anticrossings in the electron part of the spectrum that continue into the hole part, which are related to the higher energy band of the four-band model. At these anticrossings, we find a very sharp peak in the probability density close to the impurity, reminiscent for the fall-to-center phenomenon. In this paper, these peculiar and interesting effects are studied for different magnetic field, interlayer coupling, and bias potential strengths.
\end{abstract}

DOI: 10.1103/PhysRevB.98.115406

\section{INTRODUCTION}

Graphene is a stable two-dimensional (2D) material with very interesting properties such as high thermal and electrical conductivity [1]. Its quasiparticles are described by a $2 \mathrm{D}$ Dirac-Weyl equation with a linear spectrum at low energies. This linear, relativisticlike spectrum together with the zero band gap has very fundamental implications when a Coulomb impurity is added to the graphene system. When the charge of a point Coulomb impurity exceeds a critical value, two inextricably intertwined phenomena will occur [2-6]: (i) the wave function will oscillate in space with infinitely increasing frequency near the impurity, physically corresponding with an electron falling to the center of the impurity. A complete fall-to-center can only be prevented by regularizing the problem through, e.g., the introduction of a cutoff [7]. Note that after this regularization, the fall-to-center physics manifests itself as a very sharp peak in the probability density located very close to the impurity, (ii) after this regularization, the electronic energy levels will dive into the negative hole continuum and hybridize with it; this diving into the continuum is called the supercritical instability phenomenon, which is also referred to as atomic collapse. After entering the continuum, the bound state turns into a resonance corresponding to a quasibound state [6]. This resonance is observed as a peak in the local density of states (LDOS) at the position of the impurity. In the presence of external confinement, for example, with a magnetic field or in a quantum dot, the peak in the LDOS is replaced by a series of anticrossings between discrete

\footnotetext{
*robbe.vanpottelberge@uantwerpen.be

†zareniam@missouri.edu

francois.peeters@uantwerpen.be
}

confined hole states [8-10]. When confinement is decreased (by making the quantum dot larger or decreasing the magnetic field) these anticrossings merge into a resonance level [9]. The fall-to-center phenomenon is a direct consequence of the particular scaling of the potential and kinetic energy with distance to the impurity, making a quasibound state on the impurity energetically favorable [11]. The supercritical stability phenomenon, on the other hand, occurs in every system with nonlinear conduction and valence bands for sufficient large strength of the Coulomb potential. It is important to realize the difference in signature of these two phenomena: while the supercritical instablity is seen as the diving of a bound state into the continuum hybridizing with it, the fall-tocenter physics is seen as a sharp peak in the electron density very close to the impurity when entering the continuum. The charge for which these phenomena occur is called the critical charge. In relativistic quantum physics with real electrons, this critical charge is so large that the above phenomena were never observed $[12,13]$. However, in graphene the effective fine structure constant $\alpha$ is much larger because the Fermi velocity is about 300 times lower than the velocity of light, giving an $\alpha \sim 1$. Recently atomic collapse has been detected in monolayer graphene (MLG) using charged dimers placed on top of graphene [14] and with charged vacancies [15].

$\mathrm{AB}$-stacked bilayer graphene (BLG) is another interesting 2D material that consists of two van der Waals coupled monolayers of graphene placed on top of each other with interlayer coupling $t \approx 0.377 \mathrm{eV}$ [16]. BLG has a low-energy quadratic spectrum with zero gap, which at high energy becomes linear. Because of the fundamentally different energy spectrum it is not obvious how the fall-to-center and supercritical instability phenomena manifest themselves in BLG. Previously [11], it was argued that, due to the low-energy quadratic spectrum, the fall-to-center phenomenon will not occur in BLG since the 
kinetic energy scales in a different way as the potential energy $[11,17]$. In a recent study on gapped BLG, it was shown that the supercritical instability takes place and electronic states dive into the negative continuum band, forming resonance states with increasing charge $(Z \alpha)$. However, they did not see any effect resembling the fall-to-center phenomenon [11]. However, this study was limited to the case of very small (but nonzero) values of the gap term and in the absence of any magnetic field. Furthermore, the calculations were performed within the two-band model and for $Z \alpha<0.8$. But another study claimed that for gapless BLG, the supercritical instability can already occur for arbitrary small values of the impurity strength [18]. Here, we will resolve these conflicting results by investigating the influence of a magnetic field on the spectrum of a Coulomb impurity in BLG. The effect of the magnetic field on the fall-to-center and supercritical instability phenomena will be investigated and we discuss the limit of small magnetic field. The effect of a band gap on these results will also be investigated. Essential differences between results from the two- and four-band model for BLG are pointed out.

The paper is structured as follows: in Sec. II we discuss the two different models we use in this paper. The equations for the four- and two-band model are derived and some limit solutions are given. The necessity and the effect of regularization is also discussed for these two models. In Sec. III, we study the gapless spectrum for different magnetic fields and angular momenta. In Sec. IV, we investigate the effect of the interlayer coupling, allowing us to go continuously from MLG to BLG, and the behavior for small magnetic fields. In Sec. V, we study the effect of a gap on the spectrum. The conclusions of our work are presented in Sec. VI.

\section{THE MODEL}

We employ the continuum approach and obtain the corresponding equations for both the four-band and two-band models in the presence of a Coulomb impurity and magnetic field.

\section{A. Four band model}

In an AB-stacked BLG, the Hamiltonian in the vicinity of the $K$ valley is given by [19]

$$
\mathcal{H}^{K}=\mathcal{H}_{0}^{K}+(\Delta U) \tau_{z}+V(r) I_{4},
$$

where $\Delta U$ is a gap term which can be realized through the application of an external gate, $\tau_{z}$ is the $4 \times 4$ matrix: $\left(\begin{array}{cc}I_{2} & 0 \\ 0 & -I_{2}\end{array}\right)$ with $I_{2}$ the $2 \times 2$ unit matrix, and $V(r)$ is an electrostatic potential term. $\mathcal{H}_{0}^{K}$ is the Hamiltonian for AB stacked BLG in the presence of a perpendicular magnetic field:

$$
\mathcal{H}_{0}^{K}=\left(\begin{array}{cccc}
0 & \pi & t & 0 \\
\pi^{\dagger} & 0 & 0 & 0 \\
t & 0 & 0 & \pi^{\dagger} \\
0 & 0 & \pi & 0
\end{array}\right),
$$

where

$$
\begin{aligned}
\pi= & -i \hbar v_{F} e^{i \theta}\left(\frac{\partial}{\partial r}+\frac{i}{r} \frac{\partial}{\partial \theta}\right)+e v_{F}\left[A_{r} \cos \theta-A_{\theta} \sin \theta\right. \\
& \left.+i A_{\rho} \sin \theta+i A_{\theta} \cos \theta\right]
\end{aligned}
$$

with $A_{\rho}$ and $A_{\theta}$ the radial and azimuthal components of the vector potential, respectively. Here we neglect effects due to trigonal warping [20]. For the $K^{\prime}$ valley, we have the following expression:

$$
\mathcal{H}_{0}^{K^{\prime}}=\left(\begin{array}{cccc}
0 & \pi^{\dagger} & t & 0 \\
\pi & 0 & 0 & 0 \\
t & 0 & 0 & \pi \\
0 & 0 & \pi^{\dagger} & 0
\end{array}\right),
$$

which are obtained from $\mathcal{H}_{0}^{K}$ by interchanging $\pi \rightarrow \pi^{\dagger}$. Note also that $\left(\mathcal{H}_{0}^{K}\right)^{\dagger}=\mathcal{H}_{0}^{K^{\prime}}$. The symmetric Landau gauge for the vector potential is used:

$$
\vec{A}=\left(0, \frac{B r}{2}, 0\right) .
$$

Plugging this in Eq. (3), we get

$$
\pi=-i \hbar v_{F} e^{i \theta}\left[\left(\frac{\partial}{\partial r}+\frac{i}{r} \frac{\partial}{\partial \theta}\right)-\frac{e B r}{2 \hbar}\right] .
$$

Solving the Schrödinger equation $\mathcal{H} \Psi=E \Psi$ using the Hamiltonian Eq. (1), we obtain the following set of coupled equations:

$$
\begin{gathered}
\hbar v_{F}\left[\frac{\partial}{\partial r}-\frac{(\tau m-1)}{r}-\tau \frac{e B r}{2 \hbar}\right] \phi_{B}^{\tau}=[E-V(r)] \phi_{A}^{\tau}-t \phi_{B^{\prime}}^{\tau} \\
\hbar v_{F}\left[\frac{\partial}{\partial r}+\frac{\tau m}{r}+\tau \frac{e B r}{2 \hbar}\right] \phi_{A}^{\tau}=-[E-V(r)] \phi_{B}^{\tau} \\
\hbar v_{F}\left[\frac{\partial}{\partial r}+\frac{(\tau m+1)}{r}+\tau \frac{e B r}{2 \hbar}\right] \phi_{A^{\prime}}^{\tau} \\
=[E-V(r)] \phi_{B^{\prime}}^{\tau}-t \phi_{A}^{\tau} \\
\hbar v_{F}\left[\frac{\partial}{\partial r}-\frac{\tau m}{r}-\tau \frac{e B r}{2 \hbar}\right] \phi_{B^{\prime}}^{\tau}=-[E-V(r)] \phi_{A^{\prime}}^{\tau}
\end{gathered}
$$

Here $\tau$ denotes the valley index, i.e., $\tau=+1$ for the $K$ valley and $\tau=-1$ for the $K^{\prime}$. To obtain the above equations, we made use of the circular symmetry of the problem and took the following ansatz for the $K$ and $K^{\prime}$ four-component wave functions:

$$
\begin{gathered}
\Psi^{K}(r, \theta)=\left(\begin{array}{c}
\phi_{A}^{K}(r) e^{i m \theta} \\
i \phi_{B}^{K}(r) e^{i(m-1) \theta} \\
\phi_{B^{\prime}}^{K}(r) e^{i m \theta} \\
i \phi_{A^{\prime}}^{K}(r) e^{i(m+1) \theta}
\end{array}\right), \\
\Psi^{K^{\prime}}(r, \theta)=\left(\begin{array}{c}
\phi_{A}^{K^{\prime}}(r) e^{i m \theta} \\
i \phi_{B}^{K^{\prime}}(r) e^{i(m+1) \theta} \\
\phi_{B^{\prime}}^{K^{\prime}}(r) e^{i m \theta} \\
i \phi_{A^{\prime}}^{K^{\prime}}(r) e^{i(m-1) \theta}
\end{array}\right),
\end{gathered}
$$

where $m$ is the angular momentum. Note that in BLG, the Hamiltonian does not commute with the orbital angular momentum $L_{z}$ but rather with the total angular momentum $J_{z}=$ $L_{z}+\hbar \tau_{z}+\hbar S_{z}[21]$.

Since we are considering the problem of a Coulomb impurity in the presence of a magnetic field, the electrostatic 
potential is $V(r)=-Z \alpha_{0} / r$, where $\alpha_{0}=e^{2} / 4 \pi \kappa, Z e$ is the charge of the impurity and $\kappa$ is the dielectric constant. In MLG, regularization is required when solving the problem of a Coulomb impurity for $Z \alpha>0.5$ [22]. The mathematical reason is the first-order derivatives in the Hamiltonian describing graphene and the singularity of the Coulomb potential when $r \rightarrow 0$, making the problem ill defined for $Z \alpha>$ 0.5 . This is not the case for the nonrelativistic Schrödinger equation, which is described by a Hamiltonian containing second-order derivatives and the problem is well defined for all values of the charge. Physically, the electron will undergo a complete fall to the center of the impurity for $Z \alpha>0.5$, which is reflected by an infinitely rapidly oscillating behavior of the wave function in the limit $r \rightarrow 0$. To prevent this, a cutoff of the potential is needed. Note, however, that in MLG, the fall-to-center physics persists after the regularization and manifests itself through the appearance of a sharp peak in the probability density very close to the impurity when entering the continuum. In MLG, the behavior of the components of the wave function in the limit $r \rightarrow 0$ is given by the following expression:

$$
\psi_{a}=\psi_{b}=r^{ \pm \sqrt{(m+1 / 2)^{2}-(Z \alpha)^{2}}-1 / 2} .
$$

For $m=0$, we find that when $Z \alpha<1 / 2$ only the solution with positive sign in the exponent is acceptable and the problem is solvable. However, when $Z \alpha>1 / 2$, the square root becomes imaginary (and the wave functions show infinitely fast oscillating behavior near the impurity) and both the positive and negative sign solutions are acceptable, making the problem ill defined and therefore requiring an extra boundary condition by, e.g., considering a finite size impurity. We would like to recall that for $Z \alpha>0.5$, the Hamiltonian is also non-Hermitian making the problem clearly ill defined. In this paper, we impose the regularization

$$
V(r)=-\frac{Z \alpha}{\sqrt{r^{2}+d^{2}}},
$$

which corresponds to the physical situation of a charge placed a distance $d$ from the top of the BLG sheet. Taking into account the fact that the distance from the top layer to the impurity is different from the distance of the bottom layer to the impurity, we get the following set of equations:

$$
\begin{gathered}
\hbar v_{F}\left[\frac{\partial}{\partial r}-\frac{(\tau m-1)}{r}-\tau \frac{e B r}{2 \hbar}\right] \phi_{B}^{\tau} \\
=\left[E+\frac{Z \alpha}{\sqrt{r^{2}+d_{1}^{2}}}\right] \phi_{A}^{\tau}-t \phi_{B^{\prime}}^{\tau} \\
\hbar v_{F}\left[\frac{\partial}{\partial r}+\frac{\tau m}{r}+\tau \frac{e B r}{2 \hbar}\right] \phi_{A}^{\tau} \\
=-\left[E+\frac{Z \alpha}{\sqrt{r^{2}+d_{1}^{2}}}\right] \phi_{B}^{\tau}, \\
\hbar v_{F}\left[\frac{\partial}{\partial r}+\frac{(\tau m+1)}{r}+\tau \frac{e B r}{2 \hbar}\right] \phi_{A^{\prime}}^{\tau}
\end{gathered}
$$

$$
\begin{gathered}
{\left[\frac{\partial}{\partial \rho}-\frac{(\tau m-1)}{\rho}-\frac{\rho}{2}\right] \phi_{B}^{\tau}=\left[\bar{E}+\frac{Z \alpha}{\sqrt{\rho^{2}+\bar{d}_{1}^{2}}}\right] \phi_{A}^{\tau}-\bar{t} \phi_{B^{\prime}}^{\tau},} \\
{\left[\frac{\partial}{\partial \rho}+\frac{\tau m}{\rho}+\frac{\rho}{2}\right] \phi_{A}^{\tau}=-\left[\bar{E}+\frac{Z \alpha}{\sqrt{\rho^{2}+\bar{d}_{1}^{2}}}\right] \phi_{B}^{\tau},} \\
{\left[\frac{\partial}{\partial \rho}+\frac{(1 m+1)}{x}+\frac{\rho}{2}\right] \phi_{A^{\prime}}^{\tau}=\left[\bar{E}+\frac{Z \alpha}{\sqrt{\rho^{2}+\bar{d}_{2}^{2}}}\right] \phi_{B^{\prime}}^{\tau}-\bar{t} \phi_{A}^{\tau},} \\
{\left[\frac{\partial}{\partial \rho}-\frac{\tau m}{\rho}-\frac{\rho}{2}\right] \phi_{B^{\prime}}^{\tau}=-\left[\bar{E}+\frac{Z \alpha}{\sqrt{\rho^{2}+\bar{d}_{2}^{2}}}\right] \phi_{A^{\prime}}^{\tau} .}
\end{gathered}
$$

Here $d_{1}$ is the distance from the upper layer to the impurity and $d_{2}=d_{1}+0.35 \mathrm{~nm}$ the distance from the bottom layer to the impurity. We introduce the dimensionless variable $\rho=$ $r / l_{B}$ using the magnetic length $l_{B}=\sqrt{\hbar / e B}$ as unit of length. This leads to the following set of dimensionless coupled differential equations:

In the above equations, we use $\bar{E}=E l_{B} / \hbar v_{F}, \bar{t}=$ $t l_{B} / \hbar v_{F}, \bar{d}_{1}=d_{1} / l_{B}, \bar{d}_{2}=d_{2} / l_{B}$ and $\alpha=\alpha_{0} / \hbar v_{F}$.

Lets first investigate the solution in the limit $r \rightarrow 0$. For a point size impurity $\left(d_{1}=d_{2}=0\right)$ and neglecting the magnetic field, Eqs. (15a)-(15d) can be reduced to

$$
\begin{aligned}
& -\left[\frac{\partial}{\partial \rho}+\frac{1}{\rho}\right] \frac{1}{\bar{E}+\frac{Z \alpha}{\rho}}\left[\frac{\partial}{\partial \rho}\right] \phi_{A}-\left[\bar{E}-+\frac{Z \alpha}{\rho}\right] \phi_{A}=-\bar{t} \phi_{B^{\prime}} \\
& -\left[\frac{\partial}{\partial \rho}+\frac{1}{\rho}\right] \frac{1}{\bar{E}+\frac{Z \alpha}{\rho}}\left[\frac{\partial}{\partial \rho}\right] \phi_{B^{\prime}}-\left[\bar{E}+\frac{Z \alpha}{\rho}\right] \phi_{B^{\prime}}=-\bar{t} \phi_{A} .
\end{aligned}
$$

Decoupling the above set of equations we get

$$
\begin{aligned}
& \left\{\left[\frac{\partial}{\partial \rho}+\frac{1}{\rho}\right] \frac{1}{\bar{E}+\frac{Z \alpha}{\rho}}\left[\frac{\partial}{\partial \rho}\right]+\left[\bar{E}+\frac{Z \alpha}{\rho}\right]\right\} \\
& \quad \times\left\{\left[\frac{\partial}{\partial \rho}+\frac{1}{\rho}\right] \frac{1}{\bar{E}+\frac{Z \alpha}{\rho}}\left[\frac{\partial}{\partial \rho}\right]+\left[\bar{E}+\frac{Z \alpha}{\rho}\right]\right\} \phi_{A}=\bar{t}^{2} \phi_{A},
\end{aligned}
$$


which, in the limit $\rho \rightarrow 0$, becomes

$$
\left[\frac{\rho}{Z \bar{\alpha}} \frac{\partial^{2}}{\partial \rho^{2}}+\frac{2}{Z \bar{\alpha}} \frac{\partial}{\partial \rho}+\frac{Z \bar{\alpha}}{\rho}\right]^{2} \phi_{A}=\bar{t}^{2} \phi_{A} .
$$

This problem is equivalent to the following Bessel equations:

$$
\left[\frac{\rho}{Z \alpha} \frac{\partial^{2}}{\partial \rho^{2}}+\frac{2}{Z \alpha} \frac{\partial}{\partial \rho}+\frac{Z \alpha}{\rho}\right] \phi_{A}= \pm \bar{t} \phi_{A},
$$

and the most general solution to Eq. (18) is given by

$$
\begin{aligned}
\phi_{A}= & \frac{1}{\sqrt{\rho}}\left(C_{1} I_{\sqrt{1-(2 Z \alpha)^{2}}}(\mathcal{Z} \sqrt{\rho})+D_{1} I_{-\sqrt{1-(2 Z \alpha)^{2}}}(\mathcal{Z} \sqrt{\rho})\right. \\
& \left.+C_{2} J_{\sqrt{1-(2 Z \alpha)^{2}}}(\mathcal{Z} \sqrt{\rho})+D_{2} J_{-\sqrt{1-(2 Z \alpha)^{2}}}(\mathcal{Z} \sqrt{\rho})\right)
\end{aligned}
$$

Here $\mathcal{Z}=2 \sqrt{\bar{t} Z \alpha}, I_{a}(x)$ and $J_{a}(x)$ are, respectively, the modified Bessel function and the Bessel function of the first kind of order $a . C_{1}, C_{2}, D_{1}$, and $D_{2}$ are constants. One can set $D_{1}=D_{2}=0$ when $Z \alpha<1 / 2$ and the problem is solvable. But when $Z \alpha>1 / 2$, two more solutions are required and, as in MLG, we will need an extra boundary condition to have a well-defined problem. Next to that, the solutions show infinitely fast oscillating behavior for $\rho \rightarrow 0$. Analogous to MLG, a cutoff is needed to prevent a complete fall-to-center for $Z \alpha>0.5$. This is in agreement with Ref. [17], where it was shown, using semiclassical arguments, that the fall-tocenter should occur in BLG for $Z \alpha>0.5$.

\section{B. Two-band model}

In the low-energy limit $E<<t$, one often describes the carrier dynamics in BLG using a two-band model defined by the Hamiltonian [19]:

$$
\mathcal{H}=\frac{1}{t}\left(\begin{array}{cc}
0 & \left(\pi^{\dagger}\right)^{2} \\
(\pi)^{2} & 0
\end{array}\right)+\left(\begin{array}{cc}
V(r) & 0 \\
0 & V(r)
\end{array}\right),
$$

where $\pi$ and $\pi^{\dagger}$ are given by Eq. (6). Taking the following form of the wave spinor:

$$
\Psi=\left(\begin{array}{l}
e^{i(m-1) \theta} \phi_{1}(r) \\
e^{i(m+1) \theta} \phi_{2}(r)
\end{array}\right),
$$

which we insert into the Schrödinger equation $\mathcal{H} \Psi=E \Psi$. We can remove the angular part and obtain the following set of coupled equations for the radial part:

$$
\begin{aligned}
& \frac{\partial^{2} \phi_{2}}{\partial \rho^{2}}+\left(\frac{2 m+1}{\rho^{2}}+\rho\right) \frac{\partial \phi_{2}}{\partial \rho}+\left(\frac{m^{2}-1}{\rho^{2}}+(m+1)+\frac{\rho^{2}}{4}\right) \phi_{2} \\
& =-\left(\tilde{E}+\frac{Z \tilde{\alpha}}{\rho}\right) \phi_{1}, \\
& \frac{\partial^{2} \phi_{1}}{\partial \rho^{2}}-\left(\frac{2 m-1}{\rho^{2}}+\rho\right) \frac{\partial \phi_{1}}{\partial \rho}+\left(\frac{m^{2}-1}{\rho^{2}}+(m-1)+\frac{\rho^{2}}{4}\right) \phi_{1} \\
& =-\left(\tilde{E}+\frac{Z \tilde{\alpha}}{\rho}\right) \phi_{2} .
\end{aligned}
$$

Here we introduced the dimensionless variable $\rho=r / l_{B}$ as in the four-band model. We also introduced the following dimensionless variables: $\tilde{E}=E t l_{B}^{2} / \hbar^{2} v_{F}^{2}$ and $\tilde{\alpha}=\alpha_{0} t l_{B} / \hbar^{2} v_{F}^{2}$.
Using the displaced Coulomb potential, Eq. (13), we obtain

$$
\begin{aligned}
& \frac{\partial^{2} \phi_{2}}{\partial \rho^{2}}+\left(\frac{2 m+1}{\rho^{2}}+\rho\right) \frac{\partial \phi_{2}}{\partial \rho}+\left(\frac{m^{2}-1}{\rho^{2}}+(m+1)+\frac{\rho^{2}}{4}\right) \phi_{2} \\
& =-\left(\tilde{E}+\frac{Z \tilde{\alpha}}{\sqrt{\rho^{2}+\bar{b}^{2}}}\right) \phi_{1} \\
& \frac{\partial^{2} \phi_{1}}{\partial \rho^{2}}-\left(\frac{2 m-1}{\rho^{2}}+\rho\right) \frac{\partial \phi_{1}}{\partial \rho}+\left(\frac{m^{2}-1}{\rho^{2}}+(m-1)+\frac{\rho^{2}}{4}\right) \phi_{1} \\
& =-\left(\tilde{E}+\frac{Z \tilde{\alpha}}{\sqrt{\rho^{2}+\bar{c}^{2}}}\right) \phi_{2}
\end{aligned}
$$

and defined $\bar{b}=b / l_{B}$ and $\bar{c}=(c+0.35 \mathrm{~nm}) / l_{B}$ as in the four-band model.

The two-band model leads to a Hamiltonian containing second-order derivatives and therefore no regularization is expected to be required. This can also be seen by considering the limit $\rho \rightarrow 0$ for the $m=0$ state. Considering a point impurity $\left(d_{1}=d_{2}=0\right)$ we get

$$
\begin{aligned}
& \left(\frac{\partial^{2}}{\partial \rho^{2}}+\frac{1}{\rho} \frac{\partial}{\partial \rho}-\frac{1}{\rho^{2}}\right) \phi_{2}=\left(-\tilde{E}-\frac{Z \tilde{\alpha}}{\rho}\right) \phi_{1}, \\
& \left(\frac{\partial^{2}}{\partial \rho^{2}}+\frac{1}{x} \frac{\partial}{\partial \rho}-\frac{1}{\rho^{2}}\right) \phi_{1}=\left(-\tilde{E}-\frac{Z \tilde{\alpha}}{\rho}\right) \phi_{2} .
\end{aligned}
$$

The equations above can be simplified by using the ansatz $\chi_{1}=\sqrt{\rho} \phi_{1}$ and $\chi_{2}=\sqrt{\rho} \phi_{2}$ and further decoupling to

$$
\begin{aligned}
& \left(\frac{\rho^{2}}{\partial \rho^{2}}-\frac{3}{4} \frac{1}{\rho^{2}}\right) \frac{1}{\left(-\tilde{E}-\frac{Z \tilde{\alpha}}{\rho}\right)}\left(\frac{\partial^{2}}{\partial \rho^{2}}-\frac{3}{4} \frac{1}{\rho^{2}}\right) \chi_{1} \\
& \quad=\left(-\tilde{E}-\frac{Z \tilde{\alpha}}{\rho}\right) \chi_{1} .
\end{aligned}
$$

In the limit $\rho \rightarrow 0$, Eq. (29) can be further simplified as

$$
\begin{aligned}
& \frac{\rho}{Z \tilde{\alpha}} \frac{\partial^{4} \chi_{1}}{\partial \rho^{4}}+\frac{2}{Z \tilde{\alpha}} \frac{\partial^{3} \chi_{1}}{\partial \rho^{3}}-\frac{3}{2} \frac{1}{Z \tilde{\alpha} \rho} \frac{\partial^{2} \chi_{1}}{\partial \rho^{2}}+\frac{3}{2} \frac{1}{Z \tilde{\alpha} \rho^{2}} \frac{\partial \chi_{1}}{\partial \rho} \\
&-\frac{15}{16} \frac{\chi_{1}}{Z \tilde{\alpha} \rho^{3}}-\frac{Z \tilde{\alpha}}{\rho} \chi_{1}=0 .
\end{aligned}
$$

In contrast to the four-band model, it can be clearly seen that when $\rho \rightarrow 0$ the last term in the above equation drops out and the equation does not depend on the charge $Z \tilde{\alpha}$. This indicates that the problem is solvable for all values of the charge without needing regularization and that the fall-to-center phenomenon will not occur in the two-band model.

\section{ZERO GAP BILAYER GRAPHENE}

In this section, we will solve the set of coupled fourband Eqs. (15a)-(15d) and the two-band Eqs. (25) and (26) numerically using the finite elements method.

In Fig. 1, the spectrum is shown as function of the impurity strength $Z \alpha$ for the angular quantum numbers $m=$ 0,1 and -1 with an applied magnetic field of $1 \mathrm{~T}$. We used $d_{1}=0.4 \mathrm{~nm}$ and $d_{2}=0.75 \mathrm{~nm}$ in accordance with Ref. [23]. The results of the four-band model are shown in blue while those of the two-band model are shown in opaque red. The 

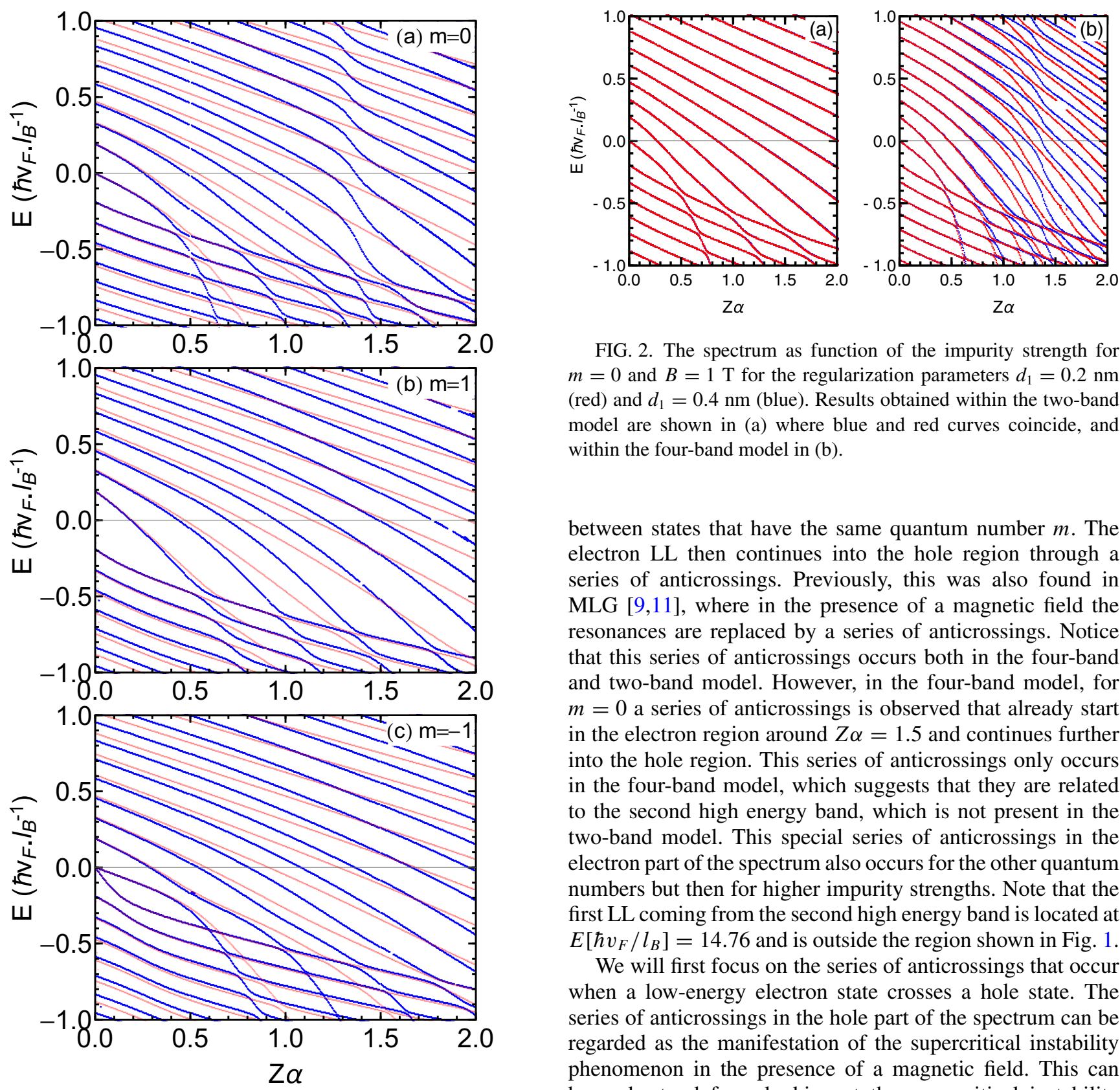

FIG. 1. The LLs as function of the impurity strength $Z \alpha$ for different values of the angular quantum number (a) $m=0$, (b) $m=1$, and (c) $m=-1$. Results from the four-band (blue curves) and two-band model (red curves) are shown. The magnetic field is $B=1 T$, giving $\hbar v_{F} / l_{B}=25.6 \mathrm{meV}$.

agreement between those two models is very good at low energies $|E|$ and small values of the impurity strength $Z \alpha$. With increasing impurity strength, the results from the fourand two-band models start to deviate, which is due to the neglect of higher order terms in the two-band Hamiltonian. In the absence of the impurity we recover the degenerate Landau levels (LLs) [24]. When the impurity strength increases, these levels start to split and the degeneracy is lifted. From Fig. 1, we see that when an electron LL approaches the first hole LL an anticrossing occurs. Such an avoided crossing occurs

FIG. 2. The spectrum as function of the impurity strength for $m=0$ and $B=1 \mathrm{~T}$ for the regularization parameters $d_{1}=0.2 \mathrm{~nm}$ (red) and $d_{1}=0.4 \mathrm{~nm}$ (blue). Results obtained within the two-band model are shown in (a) where blue and red curves coincide, and within the four-band model in (b).

between states that have the same quantum number $m$. The electron LL then continues into the hole region through a series of anticrossings. Previously, this was also found in MLG $[9,11]$, where in the presence of a magnetic field the resonances are replaced by a series of anticrossings. Notice that this series of anticrossings occurs both in the four-band and two-band model. However, in the four-band model, for $m=0$ a series of anticrossings is observed that already start in the electron region around $Z \alpha=1.5$ and continues further into the hole region. This series of anticrossings only occurs in the four-band model, which suggests that they are related to the second high energy band, which is not present in the two-band model. This special series of anticrossings in the electron part of the spectrum also occurs for the other quantum numbers but then for higher impurity strengths. Note that the first LL coming from the second high energy band is located at $E\left[\hbar v_{F} / l_{B}\right]=14.76$ and is outside the region shown in Fig. 1.

We will first focus on the series of anticrossings that occur when a low-energy electron state crosses a hole state. The series of anticrossings in the hole part of the spectrum can be regarded as the manifestation of the supercritical instability phenomenon in the presence of a magnetic field. This can be understood from looking at the supercritical instability phenomena in gapped graphene, as was discussed in Ref. [10] when the lowest electron level touches the negative continuum, the bound state will hybridize with the negative continuum forming a resonance state. In the presence of a magnetic field, the spectrum is discrete and the resonance is replaced by a series of anticrossings. In MLG, it was found that the series of anticrossings depend on the value of the angular momentum $m[9,10]$ as also seen in Fig. 1.

In Sec. II, we studied the limit $r \rightarrow 0$ solutions of both the two-band and four-band model. Here we confirm that in the four-band model, regularization is required to prevent a complete fall-to-center and to obtain solutions for $Z \alpha>0.5$ while, for the two-band model, this is not required. In Fig. 2, results are shown for two different values of the regularization parameter, shown in red $\left(d_{1}=0.2 \mathrm{~nm}\right)$ and blue $\left(d_{1}=0.4\right.$ $\mathrm{nm}$ ), for $m=0$ and $B=1 \mathrm{~T}$ both for the two-band [Fig. 2(a)] and four-band [Fig. 2(b)] model. Notice that in the two-band 

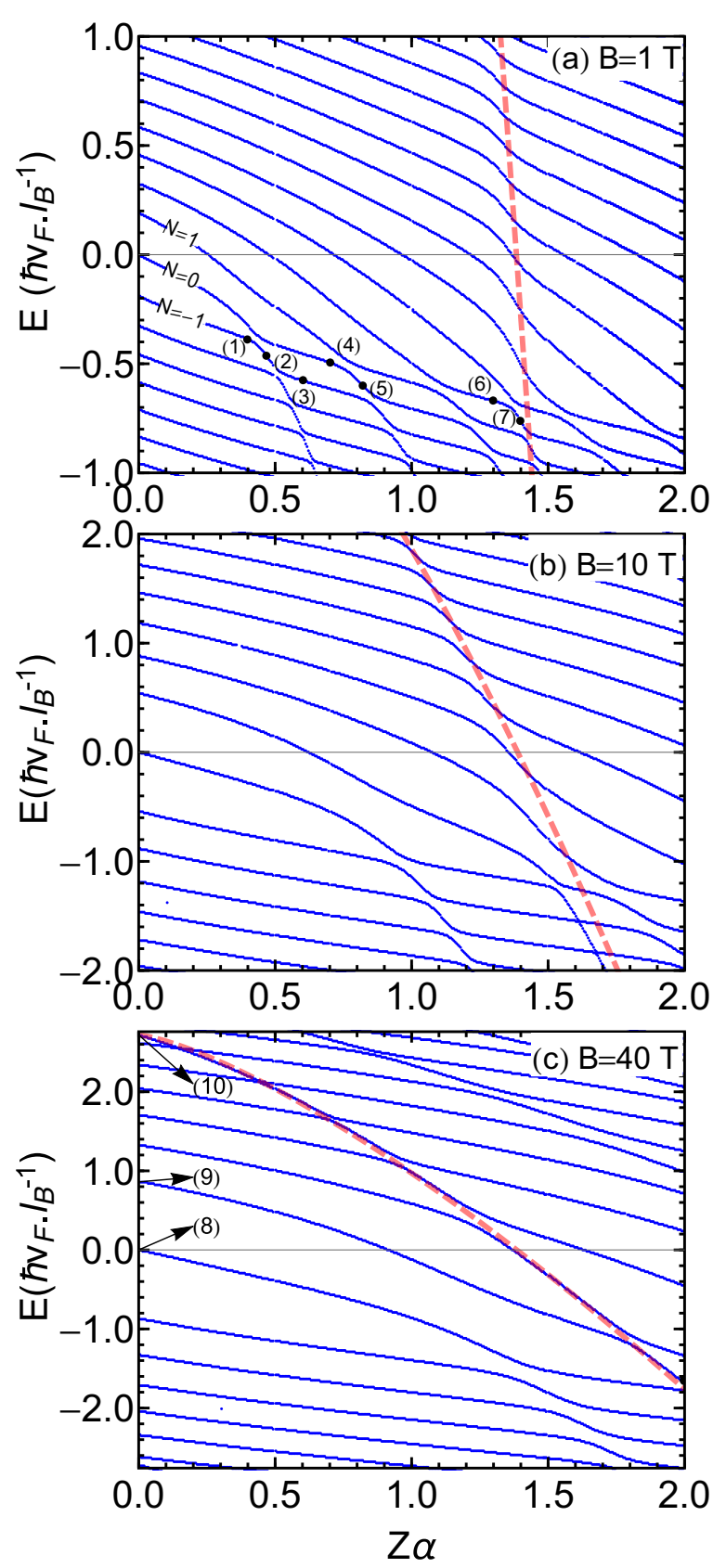

FIG. 3. The $m=0$ Landau levels as function of the impurity strength $Z \alpha$ calculated within the four-band model for a magnetic field of (a) $\mathrm{B}=1 \mathrm{~T}$, (b) $\mathrm{B}=10 \mathrm{~T}$, and (c) $\mathrm{B}=40 \mathrm{~T}$.

model, the regularization does not influence the spectrum while, in the four-band model, the energy levels and the anticrossings in particular start to depend on the regularization parameter when $Z \alpha>0.5$. Note that this transition is not abrupt at $Z \alpha=0.5$ but happens for finite value above $Z \alpha=$ 0.5 , which is due to the influence of the magnetic field.

Next we study how the spectrum depends on the magnetic field, and especially the series of anticrossings in the electron part of the spectrum. In Fig. 3, the $m=0$ spectrum as function of the impurity strength is shown for $B=1, B=10$, and $B=40$ T. It can be seen that the position of the series of anticrossings that start in the hole part of the spectrum

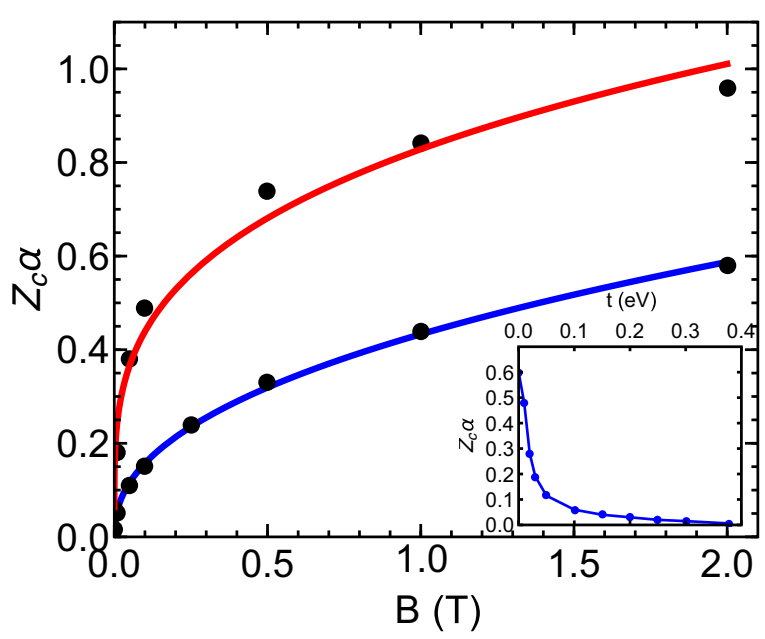

FIG. 4. The value of $Z \alpha$ for which the $N=0 \mathrm{LL}$ of $m=0$ touches the first hole LL, e.g., the position of the first anticrossing. The behavior is shown for $t=377 \mathrm{meV}$ (blue) and $t=100 \mathrm{meV}$ (red). The behavior is fitted to $Z \alpha_{c}=0.43 B^{0.44}$ (blue), $Z \alpha_{c}=$ $0.768 B^{0.3}+0.06$ (red). Inset shows the zero magnetic field critical $Z_{c} \alpha$ as function of the interlayer hopping.

depends strongly on the magnetic field. With decreasing magnetic field, the series of anticrosssings moves to lower values of the charge. In the limit $B \rightarrow 0$, the position of the first anticrossings approaches zero. This is clearly shown in Fig. 4, where the critical $Z \alpha$ for which the first anticrossing occurs (i.e., the point where the $N=0 \mathrm{LL}$ of $m=0$ touches the first hole LL) is given as function of the magnetic field (blue curve). The same is shown (red curve) for a smaller interlayer coupling $t=0.1 \mathrm{eV}$. Decreasing the coupling raises the charge for which the first anticrossing occurs. When the coupling is decreased to $t=100 \mathrm{meV}$, the critical charge tends to a finite value (see fitted function in the caption of Fig. 4). For large coupling (blue curve), we observe almost $\sqrt{B}$ dependence. This is in agreement with Ref. [11], where they observe the same functional behavior but as function of the gap. In the inset of Fig. 4 , we plot the $B=0$ critical charge $Z_{c} \alpha$ as function of the interlayer coupling $t$, demonstrating the continuous evolution from MLG to BLG. We found that for BLG, the critical charge is definitely zero. However, the exact $t$-value for which $Z_{c} \alpha$ becomes zero was difficult to determine. The reason being that we were not able to put the magnetic field exactly to zero $(B=0.001 T$ was the smallest $\mathrm{B}$-value for which our numerics was stable, and extrapolating the results to $B=0$ becomes less accurate with increasing $t$ ).

The series of anticrossings that occur in the electron part of the spectrum can be clearly seen for all values of the magnetic field. At high magnetic field, it is obvious that this series of anticrossing originates from the LLs coming from the upper band of the four-band model. In Ref. [24], it was shown that the position of the first LL coming from the upper band is given by (in units of $\hbar v_{F} / l_{B}$ ): $E=\sqrt{\bar{t}^{2}+2}$, where $\bar{t}=t l_{B} / \hbar v_{F}$. For a magnetic field of 1,10 , and $40 \mathrm{~T}$ we have, respectively, $\bar{t}=14.70,4.65$, and 2.32 and thus for $B=40$ T we have $E=2.72$, which corresponds exactly with the position of the level in Fig. 3(c) indicated by number (8). Using the exact position of the first level of the upper energy 

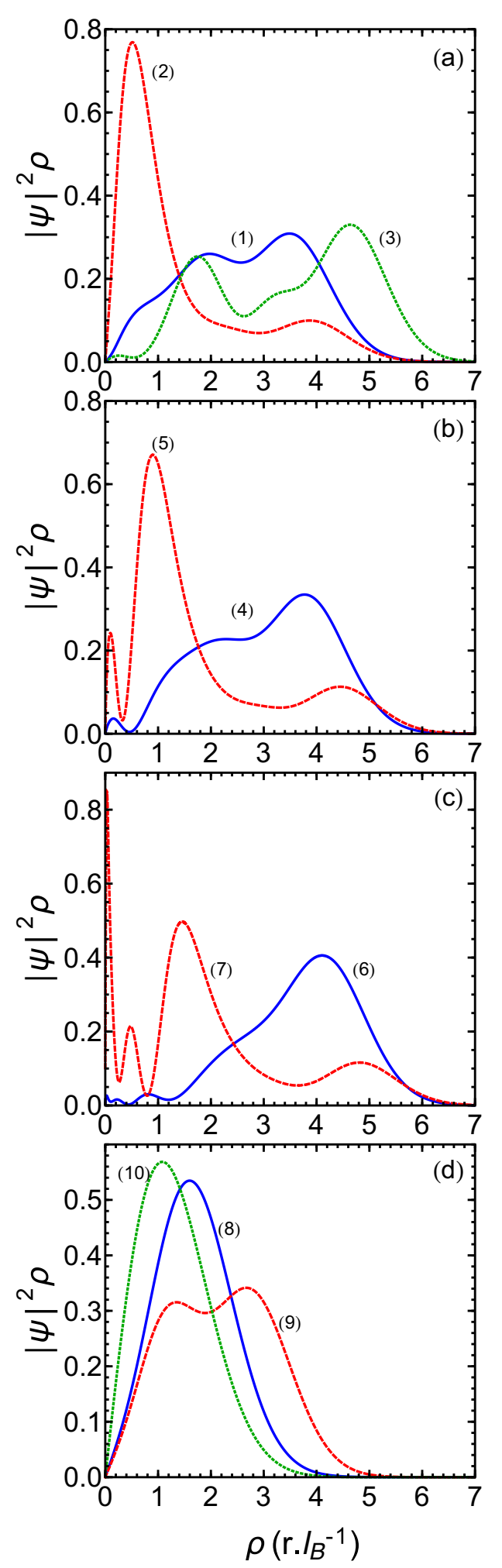

FIG. 5. The probability densities for the states marked on Fig. 2.

band, we fitted the first series of anticrossings to the function $E[\mathrm{eV}]=\sqrt{t^{2}+2\left(\hbar v_{F} / l_{B}\right)^{2}}-a(Z \alpha)^{b}$ for the three values of the magnetic field shown in Fig. 3. Fitting gives, respectively, for $B=1,10$, and $40 \mathrm{~T}:\{a, b\}=\{0.22,1.64\},\{0.24,1.47\}$, and $\{0.28,1.35\}$. The fits are shown by the dashed red curves in Fig. 3. In the case of perfect Rydberg behavior, one would

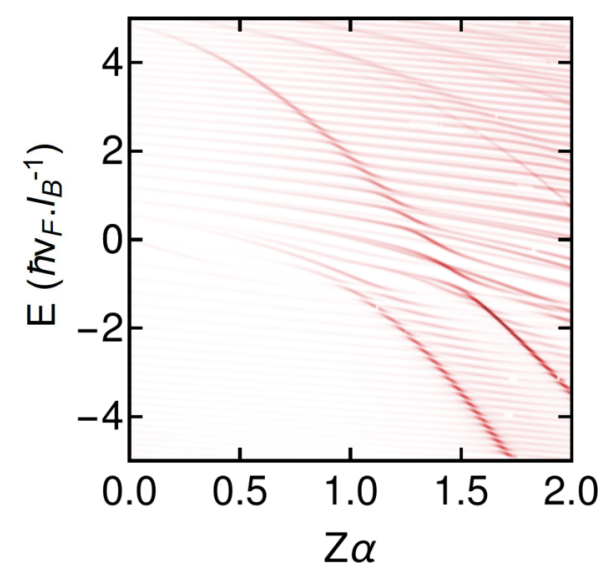

FIG. 6. Local density of states at the position of the impurity as function of the energy and impurity strength for $B=10 \mathrm{~T}$. Red in the figure corresponds to a large density of states while white corresponds to a low density of states.

find $a=2 \Delta l_{B} / \hbar v_{F}$ [25] which gives, respectively, $\{29.39,2\}$, $\{9.29,2\}$, and $\{4.64,2\}$ and is very different from our results.

In Fig. 5, we plot the probability densities for the $(Z \alpha, B)$ values indicated by the points marked on Fig. 3. The points (1), (2), and (3) correspond to the probability densities before, at, and right after an anticrossing coming from a low energy $N=0$ LL diving into the hole LLs. The points (4) and (5) are the densities before and at the anticrossing of the $N=1$ LL. The points (6) and (7) are the densities right before and at the anticrossing that is caused by a LL coming down from the upper band. Notice that at the anticrossing, the particle is more closely localized at the impurity. There is a large difference between the densities during these two different anticrossings. The density for the anticrossing of the upper band shows a very sharp peak very close to the impurity. This peak is more pronounced than for the other anticrossings. From looking at the densities in the points (8), (9), and (10), we can understand why the LLs of the upper band respond more strongly to the impurity strength since their probability densities are localized closer to the impurity.

From Fig. 3, it is seen that the LLs corresponding to the upper band have a stronger dependence on $Z \alpha$ then those from the lower band. This can be understood as follows. In the absence of the impurity and magnetic field, the upper band of BLG is separated by a gap $\Delta \approx 377 \mathrm{meV}$ from the lower band and those states can be considered to be similar to those of gapped graphene. It is well known that in gapped graphene the fall-to-center phenomenon occurs [2]. This explains why the anticrossings coming from the upper band: (i) exhibit a sharp peak very close to the presence of the impurity [see Fig. 5(c)], and (ii) why they respond more strongly to the impurity. For the low-energy anticrossings, such a sharp peak is not visible [see Figs. 5(a) and 5(b)], signifying the absence of the fall-to-center physics. This point is further supported by the fact that the anticrossings of the low-energy states are both visible in the two-band and four-band model (see Fig. 2) and by the fact that in the two-band model the fall-to-center physics is absent (as shown analytically in Sec. II).

Experimentally, the LDOS is measurable by using an STM tip. In Fig. 6, we show a density plot of the LDOS at the 


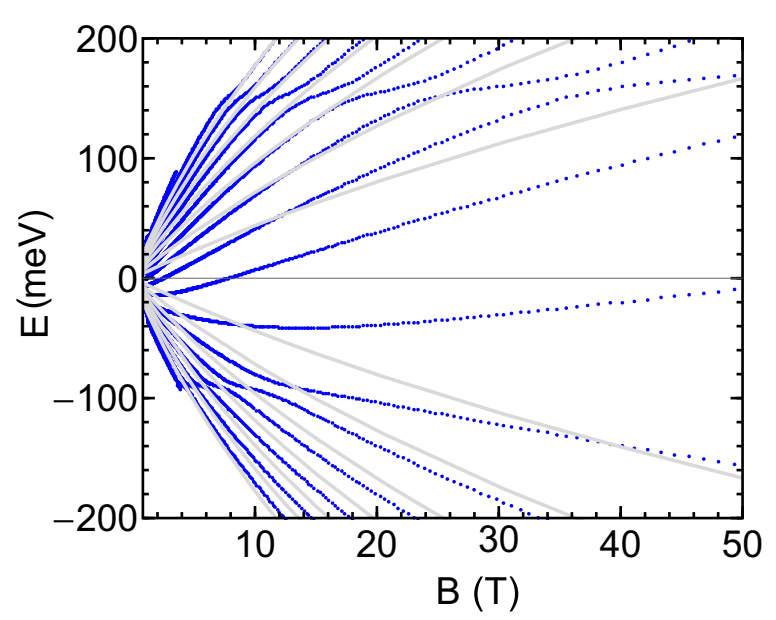

FIG. 7. The energy levels as function of the magnetic field strength for $m=0$ and $Z \alpha=1$. The gray full curves show the corresponding LLs in bilayer graphene for $Z \alpha=0$.

position of the impurity for $B=10 \mathrm{~T}$ as function of the impurity charge for a large energy range. The anticrossings originating from the upper band can be clearly seen in the figure as a large peak in the LDOS in the electron part of the spectrum. The other surrounding states coming from the lower band have a much smaller LDOS. The peak of the LDOS in the electron part of the spectrum is in sharp contrast with observations in MLG where only peaks in the hole part of the spectrum are observed. This makes these anticrossings a unique and very distinct signature. In the hole part, distinct anticrossings coming from $m=0$ states can be observed. Between those peaks, anticrossings corresponding to higher angular momentum states can already be observed.

In Fig. 7, the spectrum is shown as function of the magnetic field strength for $m=0$ and $Z \alpha=1$. The LLs without impurity are shown in gray. The energy of the LLs are pushed down by the impurity. A series of anticrossings in the electron part around $150 \mathrm{meV}$ is seen. In the hole region, similar anticrossings occur around $-90 \mathrm{meV}$. In MLG, a similar series of anticrossings in the hole region are observed and the energy position is also independent of the magnetic field. For anticrossings from the lower band, the position is determined by the position of the resonant state in the limit $B \rightarrow 0$, exactly as in MLG. This series of anticrossings corresponds to the first anticrossing seen in Fig. 3. For states coming from the upper band, the position is determined by the position of the bound state coming from the upper band in the limit $B \rightarrow 0$. This series of anticrossings corresponds to the anticrossings observed in the electron part of the spectra in Fig. 3.

\section{INFLUENCE OF THE INTERLAYER COUPLING AND SMALL MAGNETIC FIELD LIMIT}

It is interesting to investigate how the spectrum evolves as function of the interlayer coupling parameter. This gives us more insight in how the atomic collapse evolves from monolayer to BLG. This, in particular, is interesting when investigating the $B \rightarrow 0$ limit.
In Fig. 8 , the $m=0$ LL spectrum is plotted for different values of the interlayer coupling strength. In the top figures, the spectrum is given as function of the impurity strength. In the bottom pictures, the probability densities are given for the lowest $N=0 \mathrm{LL}$ (the level that starts in zero) for different values of the impurity strength. When the interlayer coupling is zero, we have two noncoupled monolayers of graphene. This can be clearly seen in the blue figure where for $Z \alpha=0$ all the levels (except the zero energy one) are double degenerate. When the charge is increased, this degeneracy is lifted and the electron levels dive in the negative energy region. When they hit the first hole LL, they anticross and the levels continuously dive in the negative hole region as a series of anticrossings. In the case of MLG, a resonance would be produced, which is replaced by a series of anticrossings when a magnetic field is present. For small value of the charge, the localization of the wave function does not change that much. However, when $Z \alpha \approx 1$, the wave function becomes localized very close to the impurity center and a sharp peak is seen accompanied with a sharp drop in the energy spectrum. Note that for zero coupling, the wave function of $N=0$ is only localized on the top layer, which is closest to the impurity. In principle, one expects that both decoupled layers are equivalent except for the difference in the distance of the impurity from the graphene sheet. However, one should keep in mind that the conserved quantity is $J_{z}$ and not $L_{z}$ and that $m=0$ for the top layer is equivalent to taking $m=-1$ when considering the bottom layer due to the $S_{z}$ term in the expression for $J_{z}$. Therefore, we will obtain an almost equivalent energy spectrum for $m=-1$ (and thus the $N=0$ level will be almost double degenerate keeping in mind that $d_{1} \neq d_{2}$ ) with the bottom and top wave functions interchanged.

When interlayer coupling is added the behavior changes qualitatively. Due to the coupling, the degeneracy at $Z \alpha=0$ is lifted and the levels hybridize. Half of the levels move down and form the LLs connected to the lower band and half of the states move up and are connected to the upper band. As discussed above, the anticrossings in the electron part of the spectrum are caused by states from the upper band. During the anticrossings, the probability densities show a very sharp peak very close to the impurity. Note that when the coupling increases, the anticrossings become stronger. The lower energy LLs still exhibit anticrossings when they hit the set of Landau hole states. However, when studying the wave functions, it can be clearly seen that the behavior of the wave functions changes with increasing coupling, see evolution between lower panels of Fig. 8. At low coupling, a sharp peak in the probability density is observed, which diminishes and eventually disappears when the coupling increases. Also the dependence of the levels on the impurity strength changes with increasing coupling: for zero coupling the lower energy LLs show a stronger dependence on the impurity strength. Increasing the coupling reduces this dependence on the impurity strength for the lower energy LLs, which can be clearly seen in Fig. 8. This change in qualitative behavior is explained by the change in energy spectrum, when the coupling is increased the spectrum changes from a linear to a quadratic one at low energies. The scaling of the kinetic energy changes from $1 / r$ to $1 / r^{2}$ close to the impurity and the effect of the Coulomb potential, which scales like $1 / r$, gets diminished. This change 

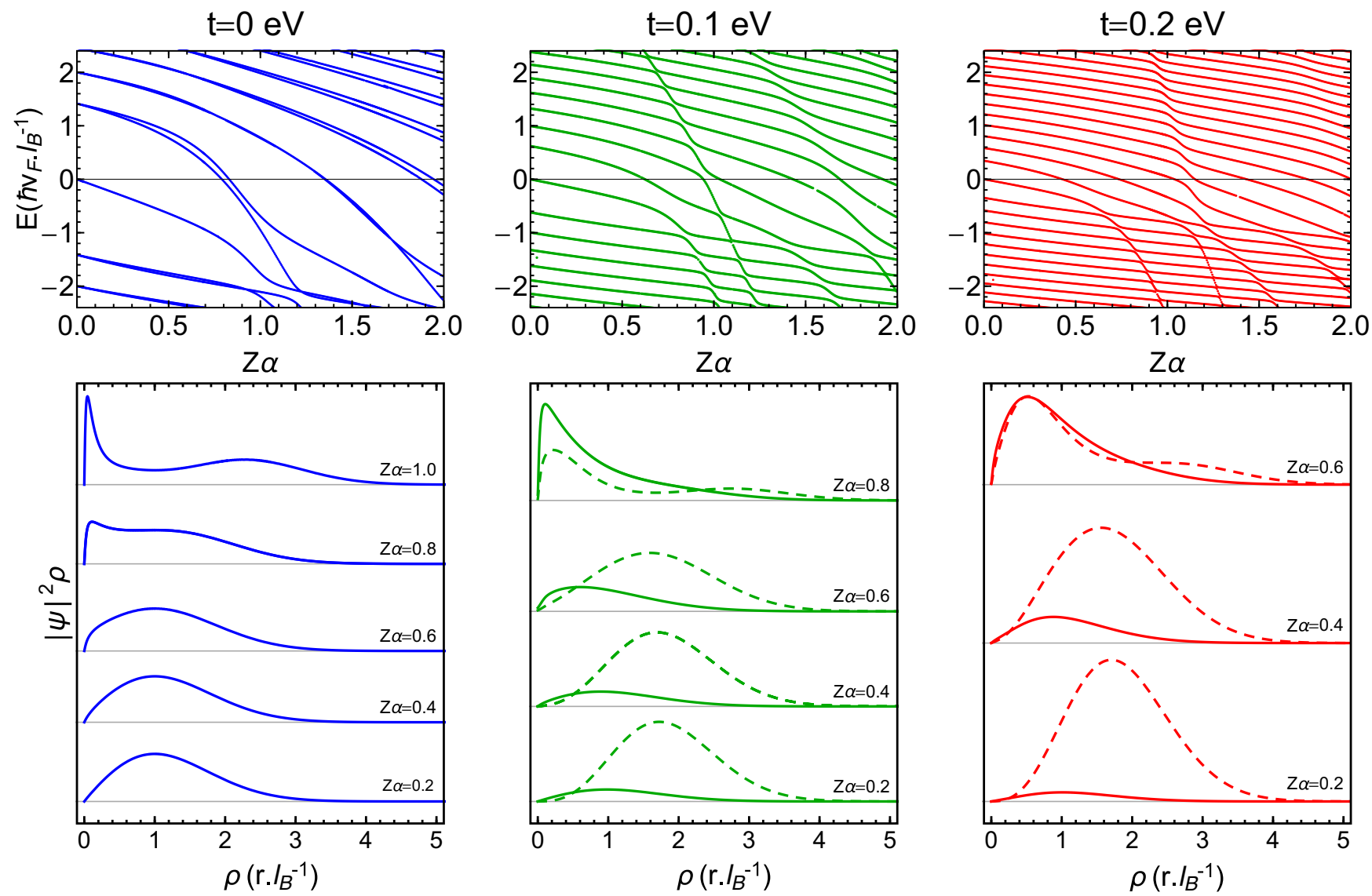

FIG. 8. Landau levels for the angular quantum number $m=0$ are shown for different values of the interlayer coupling (t). In the left figure, the coupling is zero. In the middle (right) figure, the spectrum is plotted for a coupling of $0.1(0.2) \mathrm{eV}$. In the lower panels, the probability density of the first LL $N=0$, i.e., the state starting at zero energy when $Z \alpha=0$, are shown for different values of the impurity strength. The probability density on the upper layer are shown as solid curves and on the lower layer by dashed lines. A magnetic field of 1 Tesla is applied.

of scaling of kinetic and potential energy effectively destroys the fall-to-center physics for the lower energy states.

The series of anticrossings in the electron part with increasing coupling can be clearly seen in the last two panels. The slope becomes sharper with increasing coupling. The series of anticrossings in the electron part move to higher $Z \alpha$-values with increasing coupling strength. In the middle figure of Fig. 8, the anticrossings of the second level coming from the upper band can be seen. The anticrossings in the hole spectrum seem to move to lower charges with increasing coupling. For example, for $B=1 T$ the point where the $N=0$ LL hits the first hole LL changes from $Z \alpha=1$ for $t=0$ to $Z \alpha=0.85$ for $t=0.1 \mathrm{eV}$ and $Z \alpha=0.65$ for $t=0.2$ and $Z \alpha=0.48$ for BLG.

It is also interesting to study the LDOS at smaller magnetic fields since this will provide us with information on the $B \rightarrow$ 0 limit. In Fig. 9(a), we plot the LDOS for $B=1 \mathrm{~T}$. The states coming from the upper band can be clearly seen as a sharp peak in the LDOS and should be a clear signature to look for in experiments. The anticrossings in the hole part of the spectrum coming from the low-energy states start to form a peak in the LDOS, corresponding to the quasibound state in the $B=0$ case. By comparing with Fig. 1, it is clear that the sharp anticrossings correspond to the $m=0$ states. Between the sharper peaks, two broader peaks coming from the $m=-1$ and $m=1$ states can be observed. To show this more clearly, a close-up of those peaks is shown in Fig. 9(b), note that the second $m=0$ state corresponds to one with a higher quantum number. The peak coming from the upper band exhibits clear different $Z \alpha$-dependence in the negative continuum as compared to the peaks coming from the lower band. The formation of these peaks in the LDOS is similar to MLG [9] but with one important difference: in MLG, these peaks show up for larger charges. For example, for $B=1 \mathrm{~T}$, the first peak shows up around $Z \alpha=1$ [9] while in the case of BLG the first peak already shows up around $Z \alpha=0.5$. This is shown in Fig. 10, where the LDOS for MLG is plotted for $B=1 \mathrm{~T}$. Notice the clear absence of states coming from the upper band in MLG and how the resonances emerges from the anticrossings.

\section{GAPPED BILAYER GRAPHENE}

Applying a bias potential to the bilayer leads to the opening of an energy gap in the spectrum. The size of the gap is given by the following formula [26]:

$$
E_{g}=\frac{\Delta U t}{\sqrt{4(\Delta U)^{2}+t^{2}}} .
$$



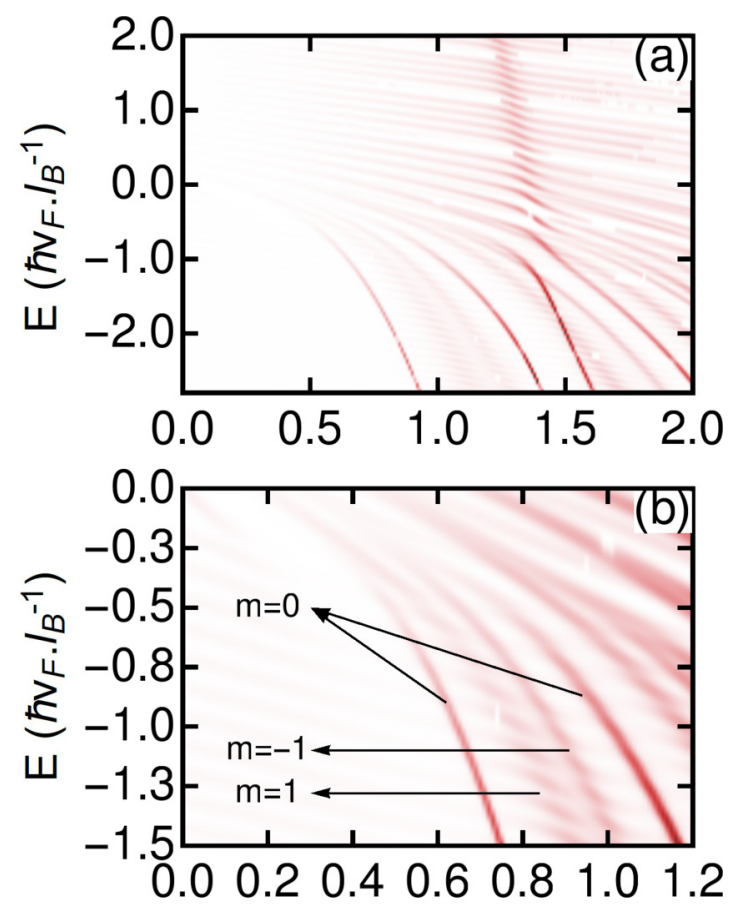

$\mathrm{Z} \alpha$

FIG. 9. (a) LDOS at the position of the impurity for $B=1 \mathrm{~T}$. (b) Close up of the peaks in the negative energy region.

Here $\Delta U$ is the applied bias voltage and $t$ the interlayer coupling parameter. From this, it can be seen that the gap in the spectrum will always be smaller than the applied bias voltage and, for high voltages, this difference will increase rapidly.

It is not immediately clear how such a gap opening will influence the impurity states. Please note that this situation is more close to the original atomic collapse problem but now in $2 \mathrm{D}[27,28]$. When a gap is present in BLG, impurity states will appear inside this gap. This situation was studied in Ref. [10] for zero magnetic field. Without a magnetic field, the spectrum

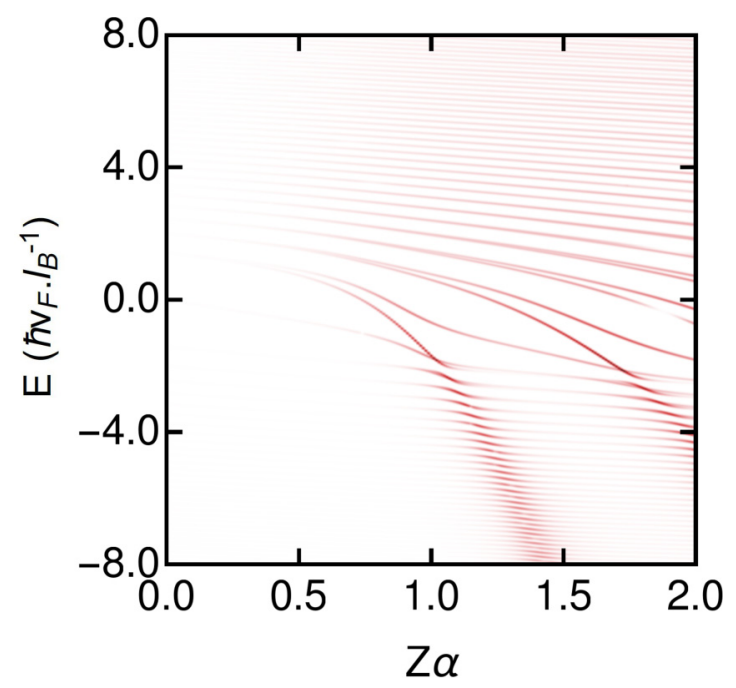

FIG. 10. Same as in Fig. 9 but for monolayer graphene.
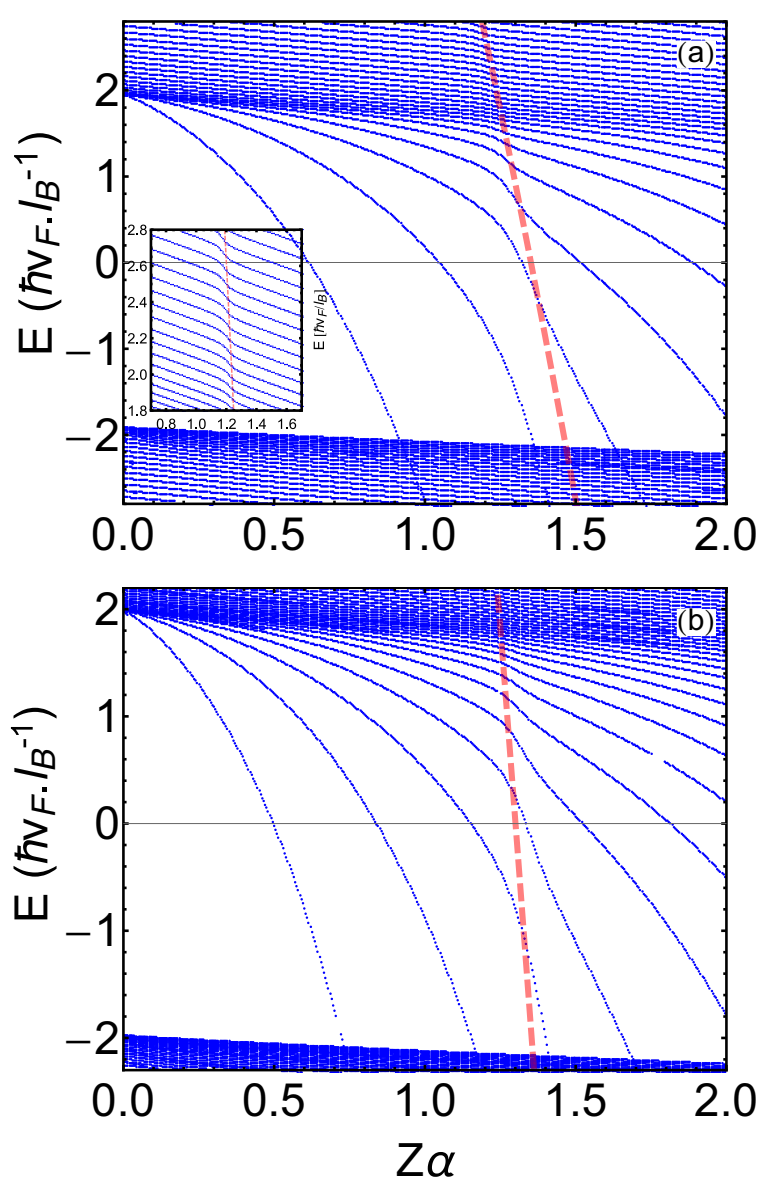

FIG. 11. (a) Spectrum as function of the Coulomb charge for a magnetic field of $1 \mathrm{~T}$ and a gap of $50 \mathrm{meV}$. A close-up of the anticrossings in the electron part seen between $Z \alpha=1$ and $Z \alpha=$ 1.5 is shown in the inset. (b) The same but now for a magnetic field of $0.25 \mathrm{~T}$ and a gap of $25 \mathrm{meV}$. Only results for angular quantum number $m=0$ are shown.

above the gap is continuous; this means that when a bound state from inside the gap enters the negative hole continuum the bound state will transform into a resonant state. This is very similar to monolayer gapped graphene.

In Fig. 11(a), the spectrum is shown for a bias potential $\Delta U=50 \mathrm{meV}$ and a magnetic field of $1 \mathrm{~T}$. In Fig. 11(b), the gap $\Delta U=25 \mathrm{meV}$ is taken twice smaller but this time we took a smaller magnetic field of $0.25 \mathrm{~T}$. In Fig. 11(a), a thick band of LLs above the gap can be seen. When the charge is increased, the lowest LLs enter the gap region and descend toward the negative band of LLs. Exactly as in the case without a gap, a series of anticrossings are seen. The series of anticrossings coming from the upper band in the electron spectrum can still be seen between $Z \alpha=1$ and $Z \alpha=1.5$. In the inset of Fig. 11(a), a close-up of these anticrossings is shown. This series of anticrossings also influences the states inside the gap region. It can be clearly seen that only higher states are affected. In Fig. 11(b), the series of anticrossings is still visible. In Ref. [11], they investigated an impurity in gapped graphene without magnetic field but they only showed the spectrum up to a charge of $Z \alpha=0.8$ and were therefore not able to observe this series of anticrossings. When an 


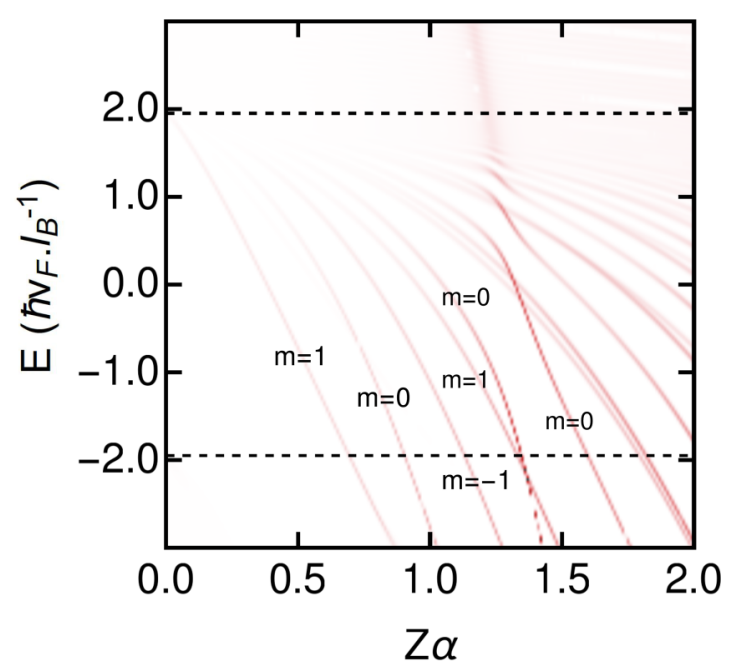

FIG. 12. LDOS at the impurity site for the parameters used in Fig. 11(a). The gap edges are given by the dashed lines.

impurity state from the gap touches a hole LL, exactly as in the gapless case, an anticrossing will occur.

For the low-energy states, the application of a bias potential opens up a gap. Because of this, the impurity-modified LLs are allowed to sink into the gap, forming true bound states. This behavior is of course consistent with the gapped case studied in Ref. [11]. The supercritical instability still occurs for these low-energy states when they touch the negative energy band of LLs. The series of anticrossings in the electron part of the spectrum still persists in the spectrum as shown clearly in the inset of Fig. 11(a). To guide the eye, the anticrossings in the electron part are denoted by an opaque red dashed line in Fig. 11, we used the same fitting formula as on page 6 with the parameters $\{a, b\}=\{0.34,1.64\}$ for Fig. 11 (a) and $\{a, b\}=\{1.47,1.64\}$ for Fig. 11(b). The lowenergy states still enter the continuum after they entered the gap region, which was also found in Ref. [11], and the series of anticrossings in the electron part remain visible showing a persistence of the effects discussed in the gapless case.

In Fig. 12, we show the LDOS at the impurity for the same paramters as in Fig. 11(a). Note that more states are visible compared to Fig. 11(a) where we show only the $m=0$ states. We indicated the angular quantum number of the states. Note that the first state entering the gap is an $m=1$ state, which is consistent with the results shown in Ref. [11]. In this figure, the anticrossings from the upper band are still visible as a higher intensity peak in the electron continuum. When the anticrossings enter the gap, region they influence the bound states inside the gap, which can be seen as an increase in strength of the LDOS. This shows that the behavior remnant of the fall-to-center phenomenon is still visible when a gap is added to the spectrum.

\section{CONCLUSIONS}

In this paper, we investigated the spectrum of a Coulomb impurity in BLG in the presence of a perpendicular magnetic field. A number of interesting properties and effects were found, which we will summarize point by point:

(1) As function of the impurity strength, the degenerate LLs split in a similar fashion as in MLG [23].
(2) The validity of the two-band model when an impurity is present is limited. Although there is very good agreement for low values of the charge, the two-band and four-band model results deviate rapidly with increasing $Z \alpha$. Anticrossings that occur when a low-energy LL touches the hole LLs are found both in the two- and four-band model. However, additional anticrossings are found in the electron part of the spectrum in the four-band model, which are absent in the two-band model. In Ref. [11], it was found that the results from the two band and four-band model agree very well for gapped BLG. Those authors limited themselves to very small values of the gap in the absence of magnetic field and to the lowest energy level for which, indeed, the two-band model gives a good description.

(3) By studying analytical solutions, we showed that in the four-band model regularization is required to prevent a complete fall-to-center for $Z \alpha>0.5$. Beyond this charge value, the four-band spectrum becomes sensitive to regularization. In the two-band model, such regularization is not required. As a consequence, the fall-to-center physics is not present in the two-band model.

(4) Anticrossings in the electron part of the spectrum are found only in the four-band model. These anticrossings were attributed to states of the second upper band. The probability density showed a very sharp peak close to the impurity at the anticrossing, being a signature of the so called fall-to-center physics. This is confirmed by the fact that the upper band is essentially similar as the band in gapped graphene, which was found to exhibit the fall-to-center physics. These series of anticrossings that dive sharply in the continuum should be clearly visible when measuring the LDOS.

(5) Anticrossings in the negative energy part of the spectrum are observed, which are manifestations of the supercritical instability phenomenon, similar as in MLG. By studying the LDOS, we showed that for smaller magnetic fields the anticrossings start to form peaks in the LDOS analogous to MLG [9]. These peaks should be clearly visible in experiments.

(6) Adding a finite gap to the system shows a persistence of the effects seen in the gapless case. Low-energy states can enter the gap and dive into the continuum at higher $Z \alpha$ values. The anticrossings in the electron part of the spectrum persist regardless of the gap.

From this study, we can conclude that the effect of a charged impurity in BLG has a dual nature. On the one hand, we have low-energy states that dive toward the negative energy states forming a distinct series of anticrossings. By studying these anticrossings and comparing with the two-band model, we showed that these low-energy states do not exhibit fall-to-center physics. However, on the other hand, we observe states coming from the upper band that do exhibit fall-tocenter physics. From this we can conclude that BLG does exhibit fall-to-center physics that can only be captured by the four-band model.

\section{ACKNOWLEDGMENTS}

We thank Matthias Van der Donck and Ben Van Duppen for fruitful discussions. This work was supported by the Flemish Science Foundation (FWO-Vl) and the Methusalem funding of the Flemish Government. 
[1] A. H. Castro Neto, F. Guinea, N. M. R. Peres, K. S. Novoselov, and A. K. Geim, Rev. Mod. Phys. 81, 109 (2009).

[2] V. M. Pereira, V. N. Kotov, and A. H. Castro Neto, Phys. Rev. B 78, 085101 (2008).

[3] J.-L. Zhu, C. Liu, and N. Yang, Phys. Rev. B 90, 125405 (2014).

[4] W. Greiner, B. Muller, and J. Rafelski, Quantum Electrodynamics of Strong Fields (Springer, Berlin, 1985).

[5] V. M. Pereira, J. Nilsson, and A. H. Castro Neto, Phys. Rev. Lett. 99, 166802 (2007).

[6] A. V. Shytov, M. I. Katsnelson, and L. S. Levitov, Phys. Rev. Lett. 99, 236801 (2007).

[7] A. A. Perelomov and V. S. Povov, Theor. Math. Phys. 4, 664 (1970).

[8] R. Van Pottelberge, M. Zarenia, P. Vasilopoulos, and F. M. Peeters, Phys. Rev. B 95, 245410 (2017).

[9] D. Moldovan, M. Ramezani Masir, and F. M. Peeters, 2D Mater. 5, 015017 (2017).

[10] O. O. Sobol, P. K. Pyatkovskiy, E. V. Gorbar, and V. P. Gusynin, Phys. Rev. B 94, 115409 (2016).

[11] D. O. Oriekhov, O. O. Sobol, E. V. Gorbar, and V. P. Gusynin, Phys. Rev. B 96, 165403 (2017).

[12] T. Cowan, H. Backe, M. Begemann, K. Bethge, H. Bokemeyer, H. Folger, J. S. Greenberg, H. Grein, A. Gruppe, Y. Kido, M. Kluver, D. Schwalm, J. Schweppe, K. E. Stiebing, N. Trautmann, and P. Vincent, Phys. Rev. Lett. 54, 1761 (1985).

[13] J. Schweppe, A. Gruppe, K. Bethge, H. Bokemeyer, T. Cowan, H. Folger, J. S. Greenberg, H. Grein, S. Ito, R. Schule, D. Schwalm, K. E. Stiebing, N. Trautmann, P. Vincent, and M. Waldschmidt, Phys. Rev. Lett. 51, 2261 (1983).
[14] Y. Wang, D. Wong, A. V. Shytov, V. W. Brar, S. Choi, Q. Wu, H.-Z. Tsai, W. Regan, A. Zettl, R. K. Kawakami, S. G. Louie, L. S. Levitov, and M. L. F. Crommie, Science 340, 734 (2013).

[15] J. Mao, Y. Jiang, D. Moldovan, G. Li, K. Watanabe, T. Taniguchi, M. R. Masir, F. M. Peeters, and E. Y. Andrei, Nat. Phys. 12, 545 (2016).

[16] E. McCann and M. Koshino, Rep. Prog. Phys. 76, 056503 (2013).

[17] E. B. Kolomeisky, J. P. Straley, and D. L. Ambrams, J. Phys.: Condens. Matter 28, 47LT01 (2016).

[18] B. Skinner, B. I. Shklovskii, and M. B. Voloshin, Phys. Rev. B 89, 041405(R) (2014).

[19] E. McCann and V. I. Falko, Phys. Rev. Lett. 96, 086805 (2006).

[20] R. Cote and M. Barrette, Phys. Rev. B 88, 245445 (2013).

[21] M. Zarenia, J. M. Pereira, Jr., F. M. Peeters, and G. A. Farias, Nano Lett. 9, 4088 (2009).

[22] O. V. Gamayun, E. V. Gorbar, and V. P. Gusynin, Phys. Rev. B 83, 235104 (2011).

[23] A. Luican-Mayer, M. Kharitonov, G. Li, C.-P. Lu, I. Skachko, Alem-Mar B. Gonçalves, K. Watanabe, T. Taniguchi, and E.Y. Andrei, Phys. Rev. Lett. 112, 036804 (2014).

[24] J. M. Pereira, Jr., F. M. Peeters, and P. Vasilopoulos, Phys. Rev. B 76, 115419 (2007).

[25] X. L. Yang, S. H. Guo, F. T. Chan, K. W. Wong, and W. Y. Ching, Phys. Rev. A 43, 1186 (1991).

[26] J. Milton Pereira, Jr., P. Vasilopoulos, and F. M. Peeters, Nano Lett. 7, 946 (2007).

[27] I. Pomeranchuk and Y. Smorodinsky, Sov. Phys. Usp. 9, 97 (1945).

[28] Y. Zeldovich and V. S. Popov, Sov. Phys. Usp. 14, 673 (1972). 